\title{
Pairing-Symmetry Selection in a Weakly Doped Canted Antiferromagnet on the Triangular Lattice
}

\author{
P. Wróbel and W. Suleja
}

Institute for the Low Temperature and Structure Research P.O. Box 1410, 50-950 Wrocław 2, Poland

\begin{abstract}
The mechanism of superconductivity generation by spin fluctuations in the electron doped canted antiferromagnet on the triangular lattice was analyzed. The underlying assumption is that the formation of the bound state is the prerequisite of pairing. The outcome of this analysis is also valid if an additional isotropic attraction is active but the anisotropic spin-fluctuation mediated force decides on the symmetry of the two-particle bound state. When the canted antiferromagnetic state is generated, the symmetry of the point group $C_{6 v}$ for the triangular lattice is lowered to the symmetry of $C_{3 v}$. It is demonstrated that spin fluctuations definitely favor the $p$-wave bound state, which transforms according to the $E$ representation of $C_{3 v}$. Since the inversion is not an element of $C_{3 v}$, the parity is not a good quantum number and thus the predicted paired state will be a mixture of singlet and triplet. Such a scenario may be relevant to physics of superconducting triangular cobaltates or organics.
\end{abstract}

PACS numbers: 71.10.Fd, 74.20.Mn, 75.50.Ee

\section{Introduction}

Correlated electron systems on triangular lattices have attracted much interest recently. Layered organic superconductors $\kappa$-(BEDT-TTF $)_{2} \mathrm{X}$ and the cobaltate superconductor $\mathrm{Na}_{x} \mathrm{CoO}_{2} \cdot y \mathrm{H}_{2} \mathrm{O}$ belong to this class of materials. The interest in their properties stimulated the revival of already ongoing theoretical research on some prototypical models for spins $1 / 2$ and on some models for correlated electrons defined on the triangular lattice.

The antiferromagnet on the triangular lattice is frustrated because this lattice is not bipartite. Rather surprisingly, it was demonstrated a few years ago that 
the antiferromagnet on the triangular lattice reveals long-range order which takes the form of the canted $\left(120^{\circ}\right)$ spin ordering [1-4].

As it has been shown for the $t-J$ model $(t J \mathrm{M})$ on the square lattice, if binding is strong enough it is sufficient to consider a moving hole pair bound by a chain of spin fluctuations, in order to understand the mechanism which determines the symmetry of pairing in a doped antiferromagnet (AF) [5]. This will not change if the tendency towards binding is strengthened by a supportive isotropic short-range interaction.

\section{Model}

In order to achieve the goal of understanding the mechanism selecting the pairing symmetry in the canted AF (CAF), it is sufficient to discuss the formation and propagation of a spin bipolaron (SBP) which represents the motion together of two holes in a single cloud formed by spin fluctuations. Some kind of spatially confining potential for holes is formed by that cloud. The construction of a SBP for the $t J \mathrm{M}[6]$ which we choose to analyze the doped AF on the triangular lattice will be now briefly outlined. All details can be found elsewhere [7]. The cobaltates are actually electron-doped systems. Nevertheless, the model which is used to analyze them can be translated by means of the particle-hole transformation into the hole doped version which is more convenient to analyze. The SBP is defined as the variational wave function obtained by finding, in a restricted Hilbert space spanning some basis states, the groundstate of an unperturbed Hamiltonian $H_{0}^{(t J)}$, which is a part of the full Hamiltonian for the $t J \mathrm{M}$. The restricted Hilbert space applied in the construction of a SBP associated with a pair of nearest neighbor (NN) sites consists of states obtained by allowing two holes created at these sites in the CAF state to hop. A restriction which is put on this hopping is that the holes may not follow each other along the same path, which guarantees that they behave as two particles in a potential well. The formation of this well should be attributed to the increase in the exchange energy induced by the creation of spin fluctuation in the CAF spin background during the hole motion. By definition, $H_{0}^{(t J)}$ obeys all the above formulated constraints and acts within the restricted space. The wave function $\left|\Psi_{\langle i, j\rangle}\right\rangle$ of a SBP created at a pair of NN sites $i, j$ is given by the lowest eigenstate of the unperturbed Hamiltonian.

The symmetry and the momentum of a two-hole bound state can be found at the level of a single SBP approximation by analyzing in the basis formed by SBP wave functions the non-vanishing off-diagonal matrix elements of the remaining "perturbed" part $H_{1}^{(t J)}$ of the $t J \mathrm{M}$. These matrix elements define an effective tight binding Hamiltonian which describes hopping of SBPs between different pairs of NN sites $\langle i, j\rangle$ and $\langle m, n\rangle$,

$$
H_{\mathrm{eff}}=\sum_{\langle i, j\rangle,\langle m, n\rangle} \tau_{\langle i, j\rangle\langle m, n\rangle} b_{\langle i, j\rangle}^{\dagger} b_{\langle m, n\rangle},
$$

where 


$$
\tau_{\langle i, j\rangle\langle m, n\rangle}=\left\langle\Psi_{\langle i, j\rangle}\left|H_{1}^{(t J)}\right| \Psi_{\langle m, n\rangle}\right\rangle .
$$

The operator $b_{\langle i, j\rangle}^{\dagger}\left(b_{\langle m, n\rangle}\right)$ creates (annihilates) a SBP at the pair of NN sites $i, j(m, n)$.

Due to lack of space we will not embark now on the detailed analysis of processes which contribute to $H_{\text {eff }}$. That task has been performed elsewhere [7]. Here we shall concentrate on some symmetry aspects of the problem. The processes which give rise to coherent propagation of a SBP should conserve the total spin of the system. It follows from that constraint that within our approach the effective Hamiltonian $H_{\text {eff }}$ may contain only terms generating hopping of a SBP between links, the ends of which have been initially occupied in the classical version of the CAF state by the same pair of spins for example by spins pointing into the "12 o'clock" direction and into the "8 o'clock" direction. Such sites form within the initial triangular lattice a honeycomb sublattice. An even more convincing argument which supports the statement that SBPs can move in the CAF background only within a honeycomb sublattice is based on the fact that the existence of the the CAF order induces an effective size reduction of the first Brillouin zone because the size of the elementary cell in the real space increases. Objects as SBPs, the motion of which does not destroy the underlying spin background, can propagate only in such a way so that the energy dispersion determined by that propagation reveals the periodicity of the reduced Brillouin zone. That requirement will be fulfilled if and only if SBPs move inside one of honeycomb sublattices. It turns out that the SBP propagation is to great extent governed by a process which in an oversimplified version looks as follows. One of two holes created at NN sites moves by one lattice to another site inside the same honeycomb sublattice. The second hole moves next to the site initially occupied by the first hole. It is not difficult to realize that such a process does not create defects in the spin background and that it gives rise to an effective shift of a SBP between linked bonds in the honeycomb sublattice. In the presence of the CAF state the $C_{6 v}$ symmetry of the triangular lattice is lowered to $C_{3 v}$ which is also the symmetry of honeycomb sublattices within which SBPs can propagate coherently. Thus we can use representations of $C_{3 v}$ and representations of its subgroups to classify eigenstates of the tight binding Hamiltonian (1). For the case of a single SBP, the groundstate of $H_{\text {eff }}$ appears at zero momentum, and is doubly degenerate because it transforms according to the representation $E$ of $C_{3 v}$. Since functions $x$ and $y$ transform according to that symmetry, the notion of $p$-wave can be applied to the expected bound and paired states. By applying the particle-hole transformation we may show that the two-electron groundstate of the related electron-doped version of the model transforms according to the same representation $E$ of $C_{3 v}$.

\section{Discussion and conclusions}

In order to summarize the main result obtained in this paper, we proceed now to discuss the structure of the predicted paired state obtained by solving $H_{\text {eff }}$. 
The formation of the CAF state in the triangular lattice gives rise to the symmetry breakdown of the inversion symmetry which induces mixing of singlet with triplet in the paired state. In order to show that the mixing will indeed take place in the anticipated by us two-particle bound state represented in the framework of the effective model by a single SBP state we will analyze its structure with some detail. Let us choose as basis vectors $\boldsymbol{a}_{1}$ and $\boldsymbol{a}_{2}$ in the underlying triangular lattice the vector $(1,0)$ and the vector $(1 / 2, \sqrt{3} / 2)$. We concentrate now on the electron doped case. It is clear that by the virtue of the particle-hole transformation the definition and the idea of SBPs can be also applied in the electron doped case to represent wave functions of additional electrons confined by the string effect in the vicinity of NN sites where they have been initially created. Analogously as in the hole-doped case these initial sites label SBPs. For the purpose of analyzing spin of the predicted bound state, in this part of the paper we formally treat a SBP created at a pair of NN sites $i, j$ as a product of two single spin polarons with spins antiparallel to the directions of the magnetization at those sites in the undoped CAF. After performing the Fourier transformation we obtain the representation for the first of two degenerate boundstates,

$$
\begin{aligned}
\left|\Psi_{1}\right\rangle & \sim \sum_{\boldsymbol{k}}\left[\left(\frac{3}{4}+\frac{\sqrt{3}}{4} \mathrm{i}\right)\left(\cos \boldsymbol{k} \boldsymbol{a}_{1}-\cos \boldsymbol{k} \boldsymbol{a}_{2}\right) p_{\boldsymbol{k}, \downarrow}^{\dagger} p_{-\boldsymbol{k}, \uparrow}^{\dagger}\right. \\
& +\left(\frac{\sqrt{3}}{4}-\frac{3}{4} \mathrm{i}\right)\left(\sin \boldsymbol{k} \boldsymbol{a}_{1}+\sin \boldsymbol{k} \boldsymbol{a}_{2}\right) p_{\boldsymbol{k}, \downarrow}^{\dagger} p_{-\boldsymbol{k}, \uparrow}^{\dagger} \\
& \left.-\left(\frac{\sqrt{3}}{4}+\frac{\mathrm{i}}{4}\right)\left(\sin \boldsymbol{k} \boldsymbol{a}_{1}+\sin \boldsymbol{k} \boldsymbol{a}_{2}\right) p_{\boldsymbol{k}, \downarrow}^{\dagger} p_{-\boldsymbol{k}, \downarrow}^{\dagger}+\ldots\right]|\Omega\rangle,
\end{aligned}
$$

where operators $p_{\ldots}^{\dagger}$ create single spin polarons and $|\Omega\rangle$ represents the undoped CAF which plays the role of a vacuum. Among three operator products which create two polarons with vanishing total momentum, the first creates a singlet and next two create triplets.

Before any conclusions for physical systems can be drawn from the above presented result, it should be verified by means of more elaborate methods, e.g. the exact diagonalisation.

\section{References}

[1] D.A. Huse, V. Elser, Phys. Rev. Lett. 60, 2531 (1988).

[2] R. Deutscher, H.U. Everts, Z. Phys. B 93, 77 (1993).

[3] B. Bernu, C. Lhuillier, L. Pierre, Phys. Rev. Lett. 69, 2590 (1992).

[4] B. Bernu, P. Lecheminant, C. Lhuiller, L. Pierre, Phys. Rev. B 50, 10048 (1994).

[5] P. Wróbel, R. Eder, Phys. Rev. B 58, 15160 (1998).

[6] K.A. Chao, J. Spałek, A.M. Oleś, Phys. Rev. B 18, 3453 (1978).

[7] P. Wróbel, W. Suleja, Phys. Rev. B 76, 214509 (1907). 